\title{
RELIABILITY DETERMINATION AND DIAGNOSTICS OF A MECHATRONIC SYSTEM
}

\author{
Yury Turygin', Pavol Božek², Ivan Abramov', Yury Nikitin'
}

1 Kalashnikov Izhevsk State University, Faculty of Quality Management, Department of Mechatronic Systems, Studencheskaya 7, 426069 Izhevsk, Russia, e-mail: turygin_uw@mail.ru; iva140742@gmail.com; nikitin@istu.ru

2 Slovak University of Technology, Faculty of Materials Science and Technology, Institute of Production Technologies, J. Bottu 25, 917 24 Trnava, Slovakia, e-mail: pavol.bozek@stuba.sk

Received: 2018.04.12

Accepted: 2018.05.06

Published: 2018.06.01

\begin{abstract}
In order to reduce the number and time of layups and to increase the period of effective operation of a mechatronic system it is reasonable to carry out predictive recovery works during one stoppage (repair) for a group of component parts with equal, close or multiple operation hours to the prior-to-failure (limiting) state. Such approach gives also the possibility to carry out recovery works with separate elements in parallel, which allows to reduce the system layup time and to increase the production output.
\end{abstract}

Keywords: determination, diagnostic, mechatronic system.

\section{INTRODUCTION}

One of the ways to increase the operational effectiveness of complex expensive mechatronic systems (machining centers, automated lines, paper-making machines, etc.) is reducing the costs of their maintenance and repair. Application of predictive maintenance and repair strategy seems to be prospective in this direction; it is based on the application of statistical analysis data of operation, failures and replacement of component parts.

However, application of such an approach in real conditions in order to enhance the operational process is quite difficult [19]. These difficulties appear due to the fact that operation time of a certain component machine part, distributed according to the known law, is a random value and failures occur in arbitrary time points. In practice, such a situation results in the case that a machine with a large number of component parts will often layup because of a failure or reaching the prior-to-failure state of only one element [21].

\section{PREDICTIVE REPAIR OF EQUIPMENT}

The urgency of searching and implementing more effective methods of complex systems (including mechatronic ones) maintenance and repair has been growing lately. It is explained by the fact that as the layouts are complicated and the quality of manufactured products is increased, the cost of producing the equipment and keeping its serviceable condition, i.e. its maintenance and repair (M\&R), is considerably increased.

Improvement of this or that system of M\&R is determined by the level of interaction between the objectively existing process of technical state variation of the object and the process of its operation, intended to keep the serviceability of the equipment. For example, as for the metal and woodworking equipment, chemical and other industries equipment, the mentioned interactions are considered by a periodical correction of a regulatory background of M\&R systems $[5,7]$. The expediency of such an approach is explained by a serial and mass production of goods with almost the same operation conditions 
and the possibility to more precise definition of the repair cycle parameters. This approach is not applicable for complex unique systems due to a high cost of its implementation. As regards the mechatronic systems, the maintenance and repair system, based on the execution of the assigned scope of recovery work according to preplanned time intervals or certain running hours independently on the actual state of the system, does not provide the required interaction between the mentioned processes in order to keep the assigned reliability level of objects and efficiency of their application.

Theoretical investigations and operation of mechatronic systems in our country and abroad show, that the most effective and progressive $M \& R$ system is the system of the predictive $M \& R$ in combination with applying the strategies of $M \& R$ according to running hours. A certain relativity of the system name is explained by its purposefulness $[4,7]$. In this case the modes of maintenance, scope and content of recovery works are assigned according to the actual technical state of the system.

The important condition to apply the strategies of predictive $M \& R$ of mechatronic systems effectively is ensuring the high level of the operation and repair manufacturability [20]. The main features of the operation and repair manufacturability are: controllability, accessibility, quickdetachability, interchangeability and repairability.

When designing and upgrading the unique complex mechanical systems (including mechatronic modules and systems), in order to meet the requirements of repairability and M\&R efficiency, it is necessary to keep the following principles:

- The reasonable separation of the layout by separately produced and maintained structural elements, leading to, as a rule, a considerable reduction of time and labor costs of M\&R.

- The accessibility of component parts of the equipment to maintain and repair, especially of those, determining the serviceability of the system.

- The specification and embodiment of control areas for technical condition of components parts and machines as a whole according to the accepted methods and means of technical and technological diagnostics.

- The reasonable embodiment of the parting and conjugation places in the machine, allowing to minimize the time and labor costs of assembling/disassembling and to provide the application of mechanization means when performing the M\&R.

- Designing the machine elements for the assigned lifetime with account of the accepted system of M\&R.

Therefore, the questions of applying this or that strategy of M\&R and achieving the highest operation effectiveness must be foreseen and provided at the stages of design and development.

The system of the predictive M\&R possesses certain properties of the predictive protective system. The principle of failures prevention is implemented when designing, upgrading and manufacturing by creation of the layout with the increased durability; when operating it is implemented by the assignment of predicting tolerances for the technical state parameters to be controlled. The principle of the scheduled preventive works provides the possibilities of increasing the effectiveness and quality of works and improving their organization. When the system of the predictive M\&R is applied, the diagnostics and general works are planned depending on the technical state of the equipment. The principle of providing the correspondence of the operating process to the process of varying the technical state of the equipment is implemented more completely.

The system of the predictive maintenance and repair is flexible and dynamic [22]. Strategies and modes of M\&R as applied to one and the same type of equipment can be varied depending on the growth of the average running hours of products, on performing the layout additional works, on creating the new methods and means of the technical diagnostics, and so on.

The applied strategies can be varied at different enterprises depending on their available equipment, production volume and professional skills of engineers and technicians.

\section{THE MODEL OF THE MECHATRONIC SYSTEM OPERATION}

In order to reduce the number and time of layups, to increase the interval of the effective operation of the mechatronic system, it is practically reasonable to perform preventive recovery works during one shutdown (repair) of a group of components with equal, close or multiple running hours up to the prior-to-failure (limiting) state $[11,1]$. From this point of view the model of operation can be presented as follows. 


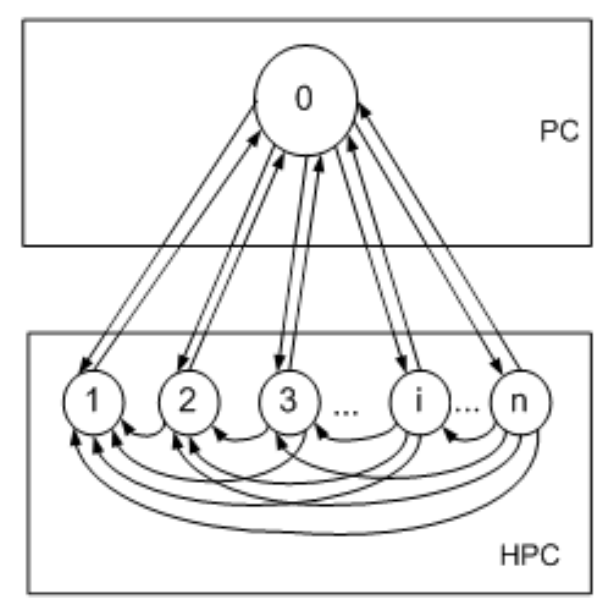

Fig. 1. The graph of states and transitions of a series system being recovered in operation (Case 1); PC the set of operable states; HPC - the set of inoperable states

\section{Case 1}

Let us consider the system, comprising $n$ component parts connected in series. The failure of any element causes the failure of the whole system. An object may have the following possible states:

" 0 " - the system is serviceable, i.e. all elements are operable;

" $1 "$ - any one element of the system is faulty, the system is inoperative;

"i" - any i elements of the system are faulty, the system is inoperative;

" $n$ " - all n elements of the system are faulty, the

system is inoperative.

The graph of states and transitions when operating the continuously working system being recovered, consisting of serially connected elements, is shown in Figure 1.

The mathematical model has been developed on the basis of the theory of semi-Markovian processes, since the laws of distribution of running hours and recoveries of component parts of the mechatronic system can have an arbitrary character, i.e. they can differ from the exponential one. Markovian properties will be exhibited only at the instants of transition from one state to the other.

When the initial state is fixed, the further behavior of the semi-Markovian process with time is fully determined by the transition probability matrix $\| p_{i j}||$ and the matrix of distribution functions $\left\|T_{i j}\right\|^{\prime \prime},\left(t_{i j}\right.$ is the holding time of the system in the state $e i$ on the stipulation that the following state of the system transition is the state $e_{0}$. At the initial time instant the process is in the state $e_{0}$. Then, according to the distribution $\left\{p_{0 j}\right\}$ the state ej of the process transition is chosen. Here, the holding time of the semi-Markovian process in the state $e_{0}$ is a random value with the distribution function $T_{0 j}(t)$. In the new state ej the process behaves in exactly the same way $[2,12]$.

The semi-Markovian process can be also assigned by means of one transition probability matrix $P[3,14]$.

$$
P=\|P(t)\|
$$

where $P(t)$ is transition possibility, i.e. the possibility of the following event: at the initial state $e_{i}$ the process will proceed by one step to the state $e_{j}$, the holding time of the process in the state $e_{i}$ will not exceed the value $t$ (the step is the time interval between adjacent transitions).

The value $P_{i j}(t)$ is related to the transition probabilities $p_{i j}$ and the distribution function $T_{i j}(t)$ by the correlation:

$$
\begin{aligned}
& P_{i j}(t)=p_{i j} \cdot T_{i j}(t), \\
& p_{i j}=\lim _{t \rightarrow \infty} P_{i j}(t)
\end{aligned}
$$

at that

$$
\sum_{j=0}^{n} p_{i j}(+\infty)=\sum_{j=0}^{n} p_{i j}=1, \quad i=0,1, \ldots, n
$$

Then one can write:

$$
T_{i j}(t)=P_{i j}(t) / p_{i j}
$$

As for the considered case with application of logical-and-probabilistic methods, the system transition probabilities from one state to the other during the time $(t, t+\Delta t)$ will be expressed as follows:

1 . If the system is in the state " 0 " at the time instant $t$, then it will stay here on stipulation that no component part will fail during the interval $(t, t+t)$. In this case the probability of such a zero transition will be determined as follows:

$$
P_{00}(\Delta t)=P_{1}(\Delta t) \cdot P_{2}(\Delta t) \ldots P_{n}(\Delta t)=\prod_{i=1}^{n} P_{i}(\Delta t)
$$

2. If the system is in the serviceable state " 0 " at the time instant $t$, then if any $i$ components fail within the interval $(t, t+\Delta t)$, it can pass to the state " $i$ " with the probability: 


$$
P_{0 i}(\Delta t)=\prod_{i=1}^{n}\left[1-p_{i}(\Delta t)\right]
$$

3. If the system is in the " $i$ "th" state, that is, it is being recovered, then it will return to the serviceable state " 0 " during the time $(t, t+\Delta t)$ with the possibility (if the repair of all $i$ components is finished):

$$
P_{i 0}(\Delta t)=\prod_{i=1}^{n} q_{i}(\Delta t)
$$

4. If the system is in the " $i^{t h \text { " }}$ state, that is, all $i$ components are being repaired, then it can stay in this state during the time $(t, t+\Delta t)$ with the possibility (if the repair of all $i$ components is not finished):

$$
P_{i i}(\Delta t)=\prod_{i=1}^{n}\left[1-q_{i}(\Delta t)\right]
$$

5. If the system is in the " $i^{t h}$ " state, that is, all $i$ components are being repaired, then it can pass to the state $j$ during the time $(t, t+\Delta t)$ with the possibility (if the recovery of all $i$ components is not finished):

$$
P_{i j}(\Delta t)=\prod_{i=1}^{m} q_{i}(\Delta t) \prod_{j=1}^{k}\left[1-q_{j}(\Delta t)\right]
$$

where $m$ is the number of recovered (repaired) components; $k$ is the number of unrecovered components.

The transition probability matrix, characterizing the state of the mechatronic system at a definite time instant, is represented in Figure 2.

\section{Case 2}

In order to reduce the order of the transition probability matrix of the model of system operation, it is proposed to apply the rule of grouping the machine components according to the states of the object, based on the principle of multiplicity of running hours. In this case, the possible states of the machine can be formulated as follows:

" 0 " - the system is serviceable, that is, all its elements are operable;

" 1 " - elements with the multiplicity 1 are faulty, the system is inoperable;

" $k$ " - elements with the multiplicity of running hours $k$ are faulty, the system is inoperable. Hence, probabilities of the system transitions from one state to the other during the time $(\mathrm{t}+$ $\Delta t)$ will be expressed as follows:

1. If the system is in the state " 0 " during the time instant $t$, then it will stay here on stipulation that no component will fail during the time interval $(t, t+\Delta t)$. The probability of such a transition will be determined by the product of probabilities of the non-failure operation of all elements of the system during the time interval $\Delta t$.

2. If at the instant $t$ the system is in the serviceable state " 0 ", then at each time interval $\Delta t$, multiple to $t_{\min }$, the system passes to the $i^{\text {th }}$ state with the probability:

$$
P_{0 i}(\Delta t)=1-\prod_{j=1}^{r} P_{i j}(\Delta t)
$$

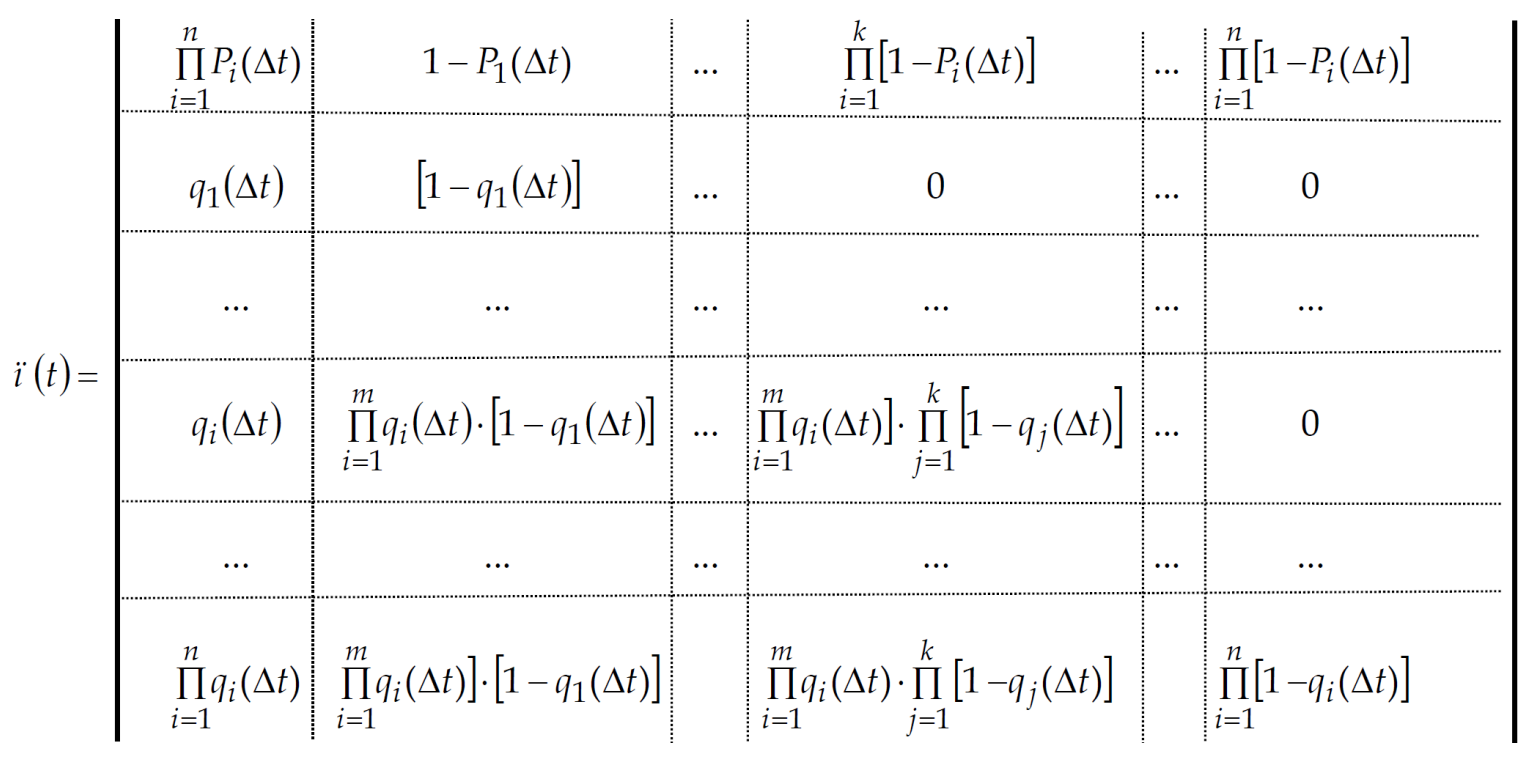

Fig. 2. The transition probability matrix 


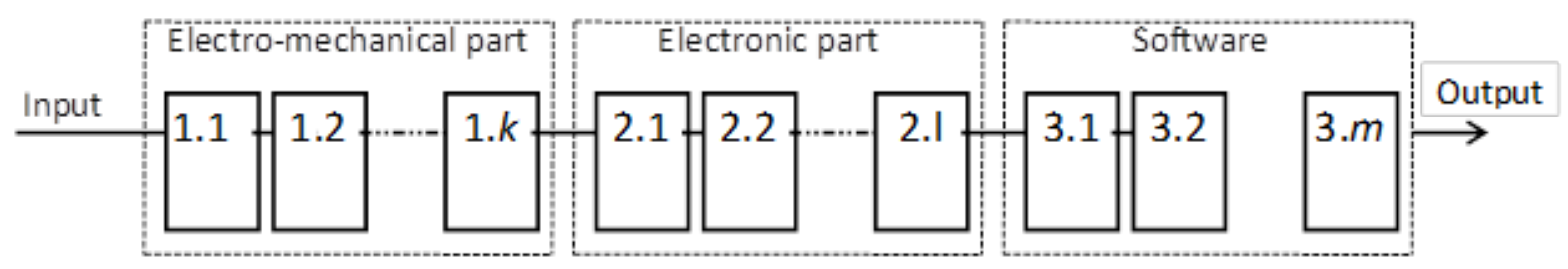

Fig. 3. The structural scheme of a mechatronic module (system) as a series system

where $j$ is the number of elements in the repaired group; $i$ is the number of the repaired group.

At the time instant $(t+\Delta t)$ recovery works in the $i^{\text {th }}$ group are started.

3. If the system is in the $i^{\text {th }}$ state, that is, repair and recovery works of the elements of the $i^{\text {th }}$ repair group are being performed, then the system will return to the serviceable state " 0 " during the time interval $(t, t+\Delta t)$ with the probability:

$$
P_{i 0}(\Delta t)=\prod_{j=1}^{r} q_{i j}(\Delta t)
$$

where $q_{j}(\Delta t)$ is the probability of recovering the component part during the time interval $\Delta t$.

4. If the system is in the $i^{\text {th }}$ state, that is, repair and recovery works of the elements of the $i^{\text {th }}$ repair group are being performed, then the system will stay in this state during the time interval $(t, t+\Delta t)$ with the probability:

$$
P_{i i}(\Delta t)=1-\prod_{j=1}^{r} q_{i j}(\Delta t)
$$

On basis of the given considerations, one can calculate the labor intensiveness of replacement or repair of components, belonging to the repair group:

$$
\tau_{i}=\sum_{j=1}^{r} Z_{j} \tau_{i j}
$$

where $Z_{j}$ is the number of workers, busy with the repair or replacement of the $j^{\text {th }}$ component part; $\tau_{j}$ is the time of replacement or repair of the $j^{\text {th }}$ component part; $r$ is the number of component parts in the repair group.

Therefore, equations (1)-(9) form the mathematical model of the system operation and they allow to solve the task of evaluating the achieved level of reliability of the mechatronic system and of determining the necessary time fund to perform repair and recovery works. The presented model creates the basis to improve substantially the organization of the M\&R and to increase the operation effectiveness of complex systems.

\section{THE RELIABILITY MODEL OF A MECHATRONIC SYSTEM AS THE TECHNOLOGICAL SYSTEM WITH SERIALLY CONNECTED ELEMENTS}

The sequence of mounting and the purpose of component parts are determined by principles of their interaction. Each functional part consists of the set of unappropriated elements. The structural scheme of such a system is shown in Figure 3.

It should be noted that the failure of any component part breaks the serviceability of the whole system, unlike many series systems, for example, of metal working automated lines, where the creation of intermediate storage devices is possible.

The basis of the reliability model of the series technological system is the study of information on failure and recovery flows. Figure 4 shows the formation of failure flows of component parts and a system as a whole.

The mechatronic system represents the system of $n$ serially connected elements. Then, owing to the assumption of the independence of elements for a series system, one can write:

$$
P_{c}(t)=\prod_{i-1}^{n} p_{i}(t)
$$

where $P_{c}$ is the probability of non-failure operation of the mechatronic system.

The average time of non-failure operation of a series system can be determined according to the formula:

$$
\overline{t_{c}}=M\left\{t_{c}\right\}=\int_{0}^{t_{k}} t \cdot f(t) \cdot d t
$$

where $M$ is the sign of the mathematical expectation; $t_{c}=\min t_{i}, 1 \leq i \leq n$ is the random time of non-failure operation of a series system.

It has been determined that during the recovery time of the failed element all the rest or certain elements of the system can be serviceable. The assumption has been taken, that during the time of 

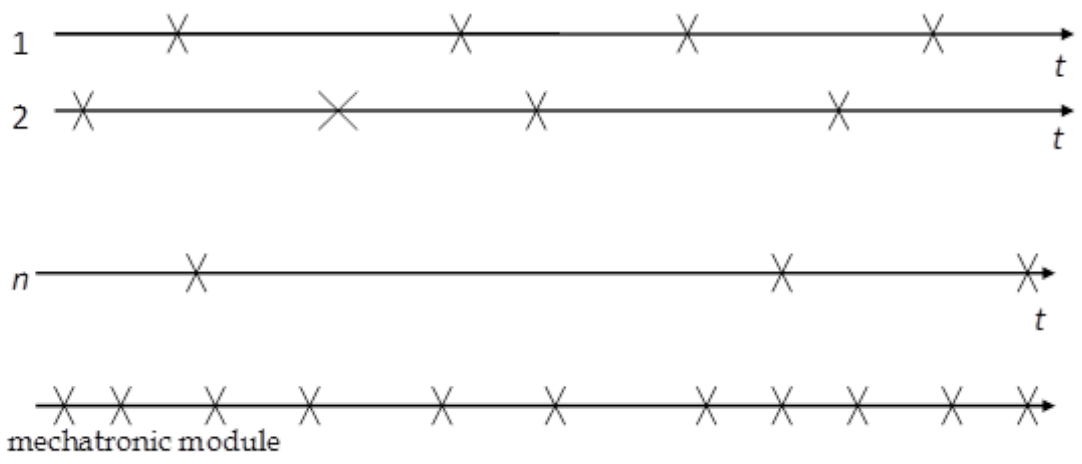

Fig. 4. Formation of the failure flow, where 1, 2, $\ldots, n$ are component parts of the system; $\times$ are instants of the failure origination

the system failures clearing (elements recovery) no variation of the reliability level takes place in all the rest elements, and that is why since the instant of the failed element recovery they begin operating as though the recovery occurs immediately. The next assumption is that owing to the great number of elements in the system, the intensity of failures of each of them constitutes a minor value of the intensity of the machine failures.

On the basis of the logical-and-probabilistic method, let us take the following probable states of the series technological system:

" 0 " - operable state;

" $i$ " - inoperable state of the system in case of fail-

ure of any one component part, $\mathrm{i}=1,2, \ldots$, $n$. ( $p_{04}$ is the probability of the element No. 4 failure during the time $\Delta t$ ).

The graph of states and transitions of the continuous action system being repaired, consisting of serially connected elements, is shown in Figure 5. As for the considered object, the failure of any element causes the failure of the whole system. A semi-Markovian process is taken as the mathematical model, because the distribution laws of running hours and recoveries of mechatronic system components can have an arbitrary character, that is, they can differ from exponential ones. Markovian processes will be displayed only at the instant of transitions from one state to the other.

With account of probable states of the system, the transition probability matrix size will be $[(n+$ $1)(n+1)]$. The matrix is stochastic, since transitions of the machine from one state to the other generate a complete group of events, the probabil-

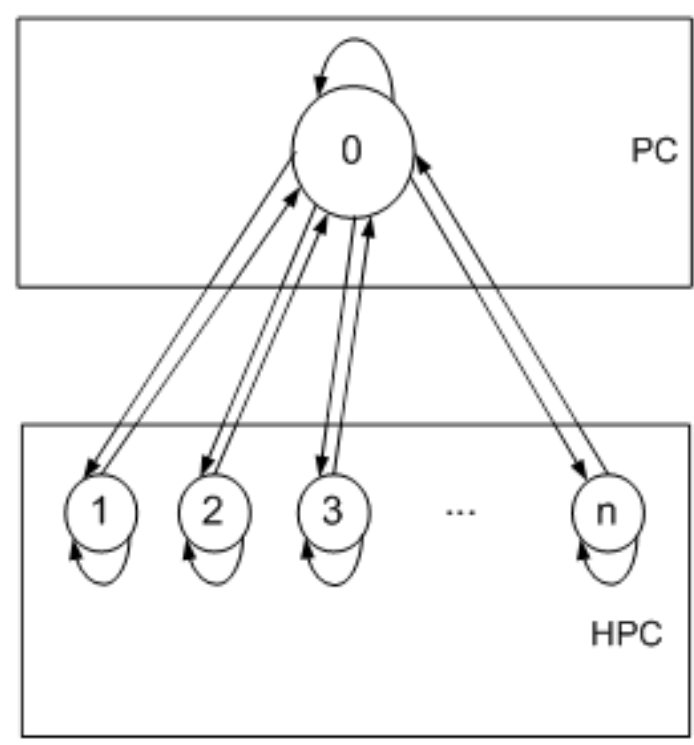

Fig. 5. The graph of states and transitions when operating the continuous action system being repaired, consisting of the serially connected elements; PC operable state; HPC - the set of inoperable states; 0 operable state; $1,2, \ldots, n$ - inoperable state because of the failure of the first, second, ..., $n^{\text {th }}$ component part of the mechatronic system

ity sum in each line must be always equal to unity. Transition probabilities during the time $(t, t+$ $\Delta t$ ) will be expressed as follows:

1. If the system is in the state " 0 " at the time instant $t$, then it will stay there on stipulation that no component part will fail during the interval $(t, t+\Delta t)$. Then the probability of such a zero transition will be expressed as follows:

$$
p_{00}(\Delta t)=p_{1}(\Delta t) \cdot p_{2}(\Delta t) \cdot \ldots \cdot p_{n}(\Delta t)=\prod_{i=1}^{n} p_{i}(\Delta t)
$$


where $p_{i}(\Delta t)$ is the probability of non-failure operation of the $i^{t h}$ element within the time interval $(t, t+\Delta t)$. 2 . If the system is in the serviceable state " 0 " at the time instant $t$, then when any component part fails within the time interval $(t, t+\Delta t)$, it will pass to the state " $i$ " with the probability:

$$
p_{o i}(\Delta t)=1-p_{i}(\Delta t)
$$

3. If the system is in the " $i^{t h}$ " state, that is, the " $i$ th" component part is being repaired, then the system will return to the serviceable state " 0 " during the time $(t, t+\Delta t)$ with the probability (if the repair of the " $i^{\text {th" }}$ component part is finished):

$$
p_{i o}(\Delta t)=q_{i}(\Delta t)
$$

where $q_{i}(\Delta t)$ is the probability of the component part recovery during the time interval $\Delta t$.

4. If the system is in the " $i^{t h}$ " state, that is, the " $i$ th" component part is being repaired, then the system can stay in this state during the time interval $(t, t+\Delta t)$ with the probability:

$$
p_{i i}(\Delta t)=1-q_{i}(\Delta t)
$$

The transition probability matrix for a system with serially connected component parts at arbitrary laws of time distribution of their running hours and recovery will have the following form (16).

One should note, that the calculation of $P_{i j}(\Delta t)$ for the time interval $\Delta t$ must be performed with account of the worked-out hours of the element and the multiplicity of the failed components replacements.

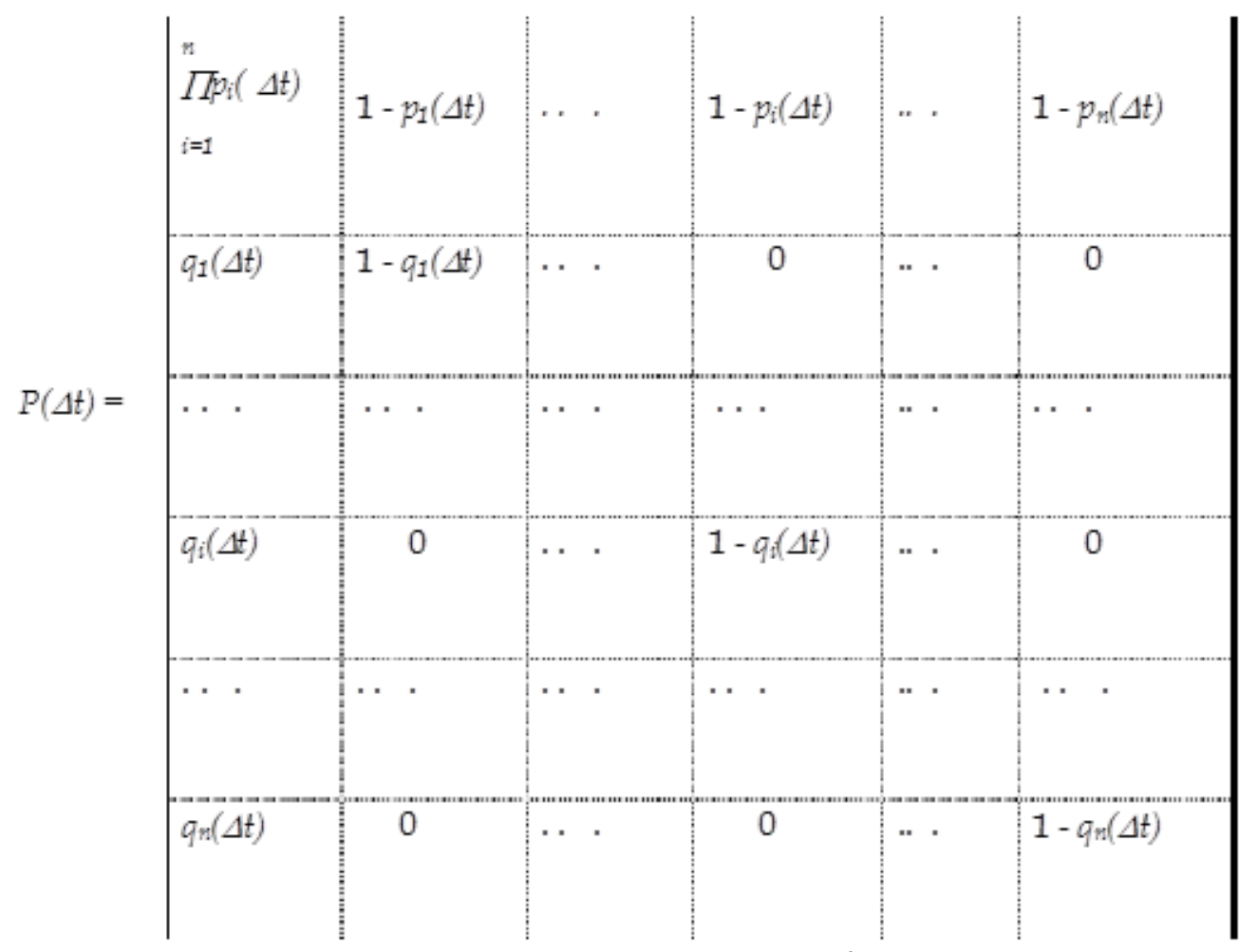

where $p_{i}(\Delta t)$ is the probability of non-failure operation of the " $i$ th" component part during the time $\Delta t$;

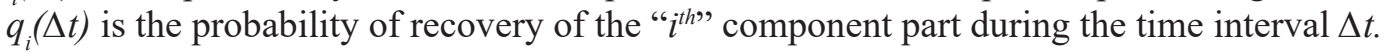

The probability of the system being in each of the states is determined according to the formula within the time interval $(t, t+\Delta t)$ :

$$
P_{i}(\Delta t)=\sum_{j=1}^{M} p_{o j} p_{i j}(\Delta t)
$$

where $i=1,2, \ldots, N$ is the number of system elements; $j=1, \ldots, M$ is the number of system states.

The relative part of the system being in the serviceable state will be equal to the availability ratio:

$$
K_{S}=P_{0}+\sum_{i=1}^{M} p_{i 2}
$$


The fund of the float time, that is, the necessary time of performing the maintenance and repair, will be:

$$
\tau=t_{k} \cdot\left(1-K_{S}\right)
$$

where $t_{k}$ is the scheduled operation life.

Composition of transition probability matrices, vectors of initial states and further determination of reliability factors implies, that the distribution laws of elements durability are known. Therefore, the development of reliability models of real machines and their component parts is possible only on the basis of information on their operation, testing and prior knowledge of analogues.

\section{DEVELOPING THE ADAPTIVE STRUCTURE OF THE REPAIR CYCLE}

\section{Construction of the mechatronic system repair schedule with account of the reliability level estimation}

Let us assume that we know the distribution laws of running hours up to the limiting state and the recovery time of each machine component. Depending on serviceability criteria, failure consequences and operation experience, the lower boundary of the limiting allowed probability of non-failure operation and parts and units recovery is assigned. According to the known distribution law and the limiting probability, the values of gamma percentile $t_{\gamma i}$ error-free running hours (until the failure) are calculated to characterize indirectly the technical state (Figure 6a) and the recovery time of each component part. The least value of the running hours is chosen among the whole range, and multiplicities of running hours are calculated according to the formula:

$$
K_{i}^{\prime}=\frac{t_{\gamma i}}{t_{\gamma \min }}, \quad \mathrm{i}=1,2, \ldots, \mathrm{n}
$$

where $t_{\gamma i}$ is the gamma percentile running hours of the $i^{\text {th }}$ element; $t_{\gamma_{\text {min }}}$ is the minimum gamma percentile running hours;

$K^{\prime}$ is the design multiplicity of running hours of the $i^{\text {th }}$ element; $n$ is the number of elements in the system.

The design value of multiplicity of gamma percentile running hours is rounded down to the least one, based on the operation experience, reducing the probability of failure (degree of risk). Then the elements are grouped according to the feature of equal multiplicity (Figure 6b). These multiplicities are $K=1 ; K=2 ; \ldots ; K=K$ and they characterize the system state. The number of elements included into each state (each group) will be different. According to such an approach, the system operation can be considered as the discrete process, that is, at discrete instants of time, multiple to $t_{y \min }$, the system passes to the state, when elements of the group $R$ will be considered as failed.

Since running hours have a random nature, then during the time interval $\Delta t$ related to the last repair, a failure or replacement of certain component parts can take place. For these parts the running hours $t_{i}$ are calculated and added to already available data, statistical processing of additional arrays is performed, which can lead either to variation of running hours $t_{\gamma i}$ of component parts, or to variation of the value $t_{\gamma \min }$. When the value $t_{\gamma \min }$ is varied, the re-calculation of running hours multiplicities is carried out. According to the obtained results, the correction of the repair graph and structure of repair groups is made. Such a correction of periodicity and $M \& R$ content is performed after the failure of any component part. Organization of $M \& R$ according to the stated principles allows considering the technical state of component parts and the system as a whole on the basis of their reliability characteristics. Periodicity and the repair content are not constant here.

During the complex system operation process the error-free running hours are of the random character. It follows that the value $t_{\gamma \text { min }}$ can be varied depending on the technical state of the element and its lifetime. In this connection the time intervals $\Delta t$ between predictive and repair and recovery works, and also the contents of repair groups (unions) are variable. The information background to implement the developed operation models is the statistical analysis of operation and repair data. Figure 7 presents the content and sequence of steps of making the decisions on repairing the mechatronic system.

At the first step the analysis of the situation takes place and the estimation of the technical state of the mechatronic system or module is performed by accumulating and processing the information. All damaged component parts are revealed, including the software. Then the identification and formulation of the problem is performed. The next step is the development of the set of alternative decisions of the problem, the level of machine-tool units damage is estimated and the decision is made on putting the mechatronic system out of operation to repair or on continuing its operation. Ideally, it is necessary 


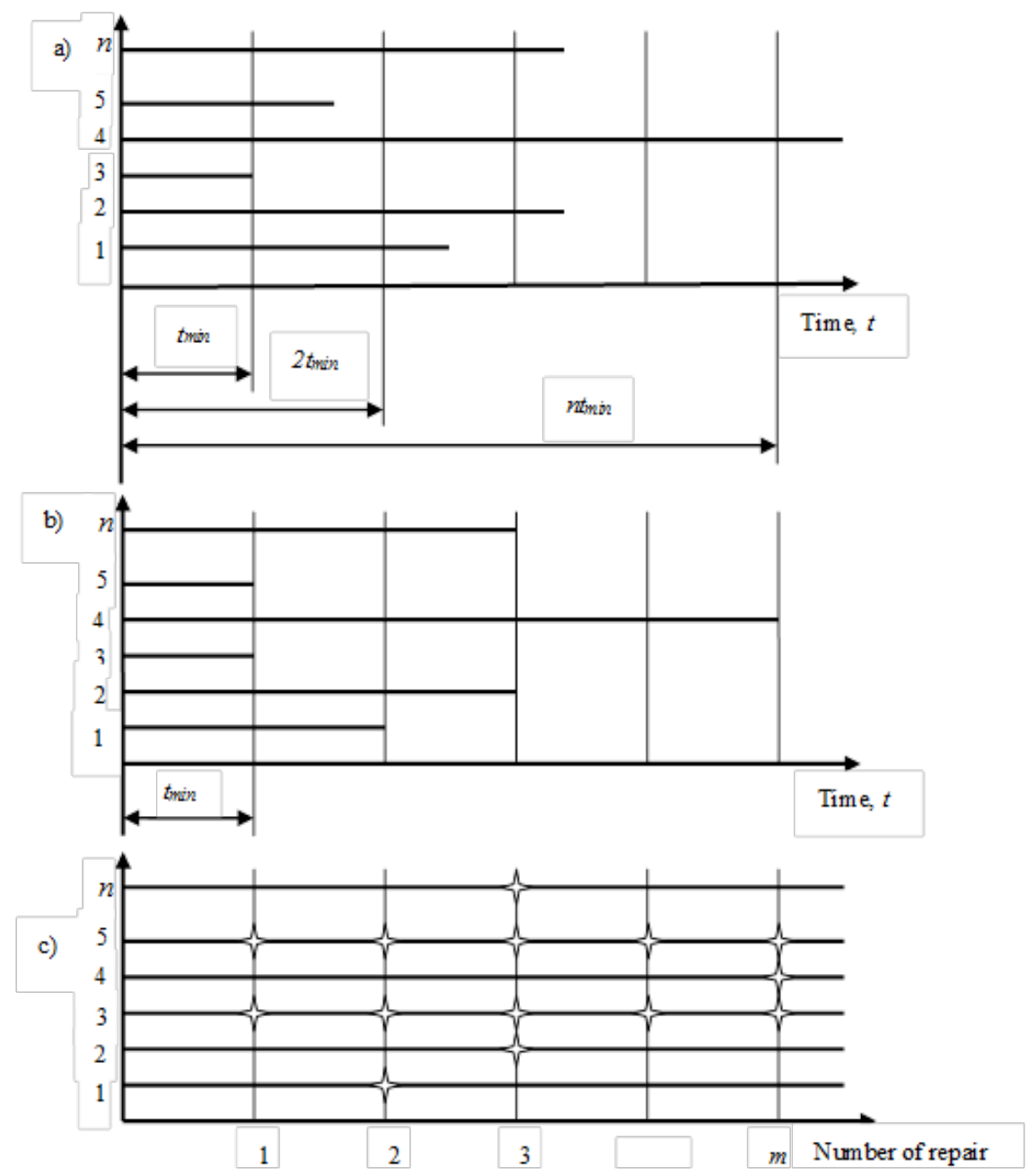

Fig. 6. To the development of the adaptive structure of the repair cycle: (a) shows the $\gamma \%$ running hours of the system component parts; (b) shows the distribution of multiple running hours of the elements; (c) shows the generation of the repair groups ( $\mathrm{m}$ is the number of the repair) where $1,2, \ldots, \mathrm{n}$ are numbers of the component (part); is the structure of the repair group

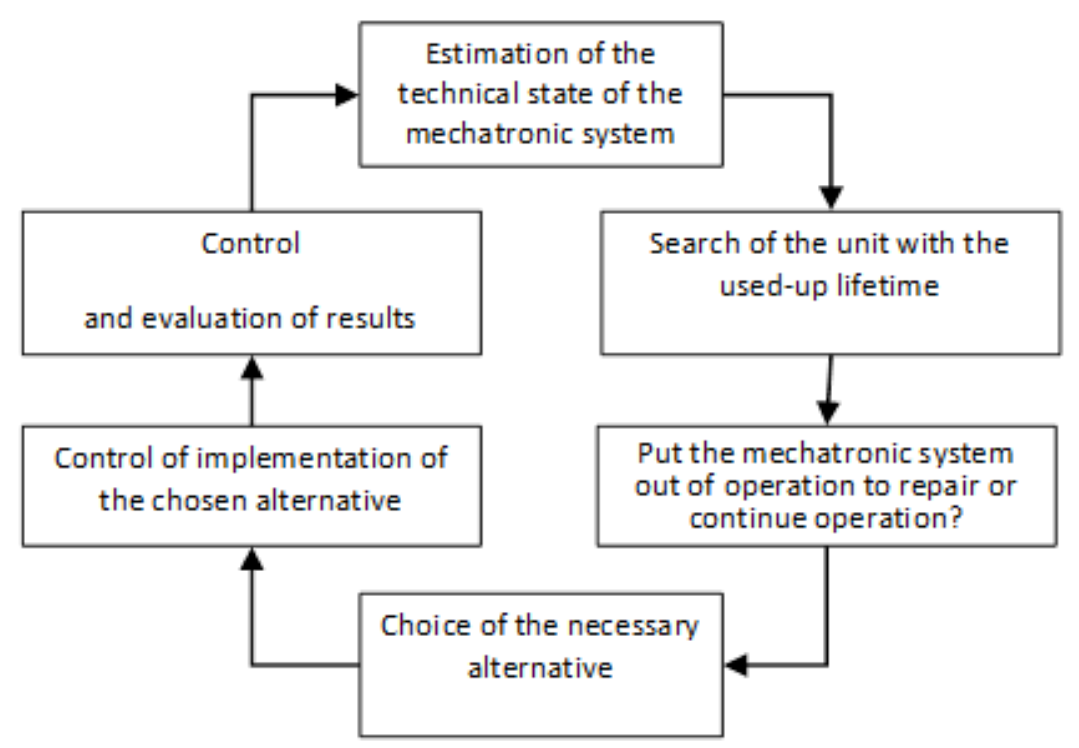

Fig. 7. Structure and sequence of steps of the decision making process of the machine-tool repair 
to detect all possible alternative decisions of the problem. Having developed the possible versions of solving the problem, they should be estimated, that is, advantages and drawbacks of each alternative should be compared and probable results of their implementation should be objectively analyzed. Further, the implementation of the taken decision is performed. And at the final step the estimation of the obtained result is carried out, serving here as the feedback function of this process. At this step the actual results are compared with the planned ones.

The proposed method allows to implement the flexible adaptive structure of the repair cycle with account of the components technical state (indirectly) for a wide variety of mechatronic systems.

\section{Development (correction) of the maintenance and repair periodicity according to the results of technical state diagnostics}

The interest to predicting the technical state and reliability of mechatronic systems has been increased recently. This fact is explained by the growth of machines manufacturability, their increased complexity, considerable losses in failures and, consequently, requirements to their reliability. The necessity of predicting is urgent at all stages of equipment development and operation: at the stage of design, during tests, when solving the tasks of diagnostic assurance, planning and operation.

The diagnostic results in mechatronic systems operation are necessary to make the decision on replacement or repair of a faulty component of the diagnostic object or a product as a whole, and on the assignment of the most reasonable periodicity and content of predictive and recovery works. The purpose of diagnostics is the estimation of the general technical state of the equipment; localization of damages; cost reduction of spare parts, materials, labor intensiveness of the equipment M\&R; changing from the scheduled M\&R to the $M \& R$ on demand and, finally, the increase of the availability factor of mechatronic systems.

The technical diagnostics in operation of machines provides the verification of their correctness, operability, functioning, search of defects and data accumulation to predict the residual life. The effect, achieved by diagnostics, is determined by the following facts: timely detection and elimination of faulty states of the machine components; elimination of unreasonable assembling/ disassembling, that keeps the technical lifetime of the diagnosed object; assurance of the whole work-out of the technical lifetime; optimal regulation of the machine operation, that increases the manufacturability and reduces the consumption of lubricants and all types of the applied energy resources; increase of the operation safety due to the possibility of frequent revisions and the control of component parts providing the safety; and application of up-to-date difficult-to-maintain but more effective component parts when developing new machines.

Application of diagnostic means regulates the machines operation, it allows to develop the automated systems of accumulating the data on the process of varying the states of diagnosed units. The latter is very important when optimizing the layouts, improving the manufacturing techniques and operation modes of newly developed machines.

The parameters of mechatronic system technical state, its separate modules and parts during the process of operation are varied from the nominal values, correspondent to technical conditions for a new equipment, up to the limiting ones. In order to justify the system of the reasonable equipment operation, including its maintenance and repair, it is necessary to know the character of variation of technical state characteristics during the period of operation.

A great number of design, production and operation factors influences the variation of technical state characteristics of a part of the unit or aggregate. The design and production factors are the quality of production, assembly, runningin, layout features and structure of separate elements and their interaction within the unit, and also physical and mechanical properties of the applied materials.

The operation factors are modes of operation (including loading modes), environmental conditions (temperature, humidity, environment pollution and so on), the intensity of the equipment operation during a shift, a day, a month, a year, etc.

From diagnostic point of view, the mathematical description of the dependence character of the technical state parameter on the running hours is of the greatest interest. It is reasonable to approximate the broken curve of the actual variation of the technical state parameter by a smooth curve. The approximating function should take into account the physics of the parameter variation, including the design and operation factors, and it should consider the character of the parameter dependence on the running hours. 
In general case the correct and faulty states of the system generate the set of its technical states. The process of diagnostics consists of separate diagnostic operations of estimating the technical state of specific component parts. Formally the result of diagnostics is the decision on the system being in one of the states within the set $E$ and on performing the activities on maintenance and repair, if needed. Having divided the set $E$ by the subsets $R$ and $Q$ of faulty states, the algorithm of organizing the process of the technical state diagnosing and its relation with the system of M\&R will have the form, presented in Figure 8.

Component parts under control can be diagnosed during the machine operation. Depending on the value of the component residual lifetime, the control can be continuous or periodical. It should be noted, that the process of obtaining the diagnosed parameter $\Pi(t)$ for a specific part or unit is the implementation of one of the diagnostic methods and it implies making the decision on estimating the technical state and residual lifetime by comparing the measured (instant) $\Pi(t)$ and limiting $\Pi_{\text {lim }}$ values of the diagnostic parameter. As for the operable components, included into the subset of the system states $R$, the value of the residual lifetime is predicted within the block $P_{0}$ on the basis of the reliability model or variation of the diagnosed parameter in operation.

When the component reaches the limiting state, it passes to the subset $Q$ and the decision is made on the terms of the damages elimination. On the basis of the taken decision on the terms of the damages elimination of the diagnosed component, repair and recovery measures (prevention or replacement of the faulty element) on eliminating the failure are taken according to the system of maintenance and repair.

The presented algorithm shows, that the technical diagnostics is necessary to estimate objectively the technical state of responsible elements of the design, it is the constituent part of the system of the predictive maintenance and repair of mechatronic systems and it provides the highest level of operational effectiveness.

In this connection the tasks of mechatronic systems diagnostic can be stated as follows: to study and establish the features (parameters) of estimating the faults and failures of the mechatronic system, its separate modules, components and parts; to develop methods and means of characterizing the fault and failure; to perform the prediction of the residual lifetime of mechatronic

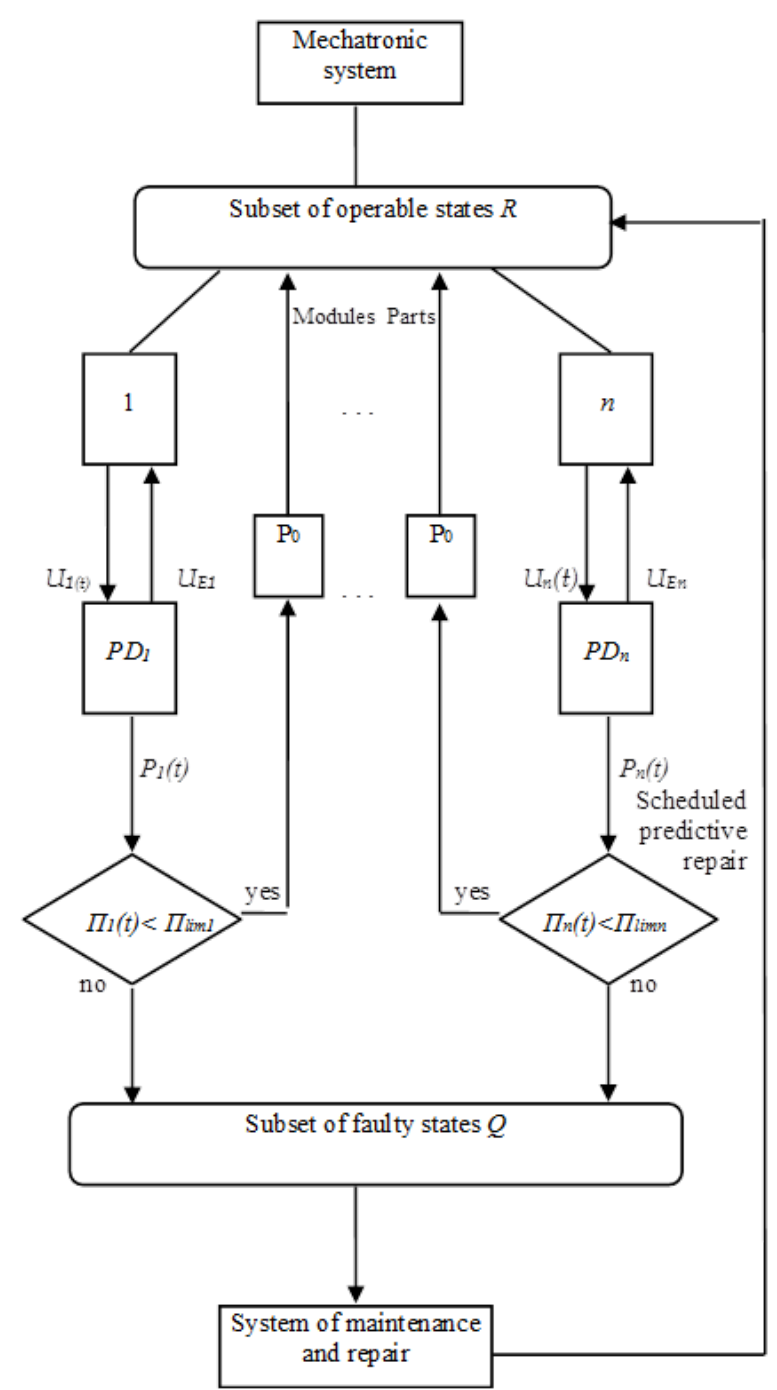

Fig. 8. Algorithm of constructing the system of maintenance and repair applying the technical state diagnostics of the mechatronic system components

modules and their components on the basis of measuring results.

The important advantage of repairs according to the technical state is a more complete application of lifetime of the equipment parts and components, possibility of predictive replacement of parts, which reached the near-to-limiting technical state, and reduction of the emergency states in the equipment operation. The effect is achieved by the layups reduction during the repair and by the increase of production output. The possibility appears to assign more reasonably the content and periodicity of repairs, to define their labor-intensiveness and to improve the preparation to the repair.

The diagnostic result can be presented by qualitative characteristics as the measured values of the diagnostic parameter and estimation "val- 
id-invalid" ("correct-faulty"), and by a quantitative value of the residual lifetime. The results of qualitative evaluation of the technical state are applied heuristically, the prediction of further behavior of the component is based on the personal experience of the expert.

In addition to the qualitative evaluation, the quantitative evaluation of the component residual lifetime provides a more profound result [12]. In order to predict the residual lifetime of components having the monotonically varying diagnostic parameter, one can apply the method, proposed in $[1,6,9,12,17]$. The mathematical description of the process of the technical state parameter variation $U(t)$ after the runningin period is based on approximation of each implementation of this process by a random function (21).

$$
U(t)=V \cdot t^{a}+\Delta P
$$

where $\Delta P$ is the parameter, characterizing the running-in of interacting surfaces; $V$ is the value, characterizing the intensity (rate) of the parameter variation; $\alpha$ is the degree index, defining the dependence of the parameter on running hours (it is established by means of a statistical processing of experimental data).

Then the average residual lifetime of the component is calculated according to the data of variation of the parameter of its technical state $U_{k}$ and running hours $t_{k}$ by the instant of control:

$$
\begin{gathered}
t_{\text {ost }}^{\text {av }}=t_{k}\left[\left(\frac{U_{p}}{U_{k}}\right)^{1 / a}-1\right] K_{t} \\
K_{t}=1+t_{M}^{\alpha}\left(\frac{U_{k}}{U_{p} \cdot t_{k}^{\alpha}}-\frac{1}{\sqrt{\alpha \cdot t_{a v}^{\alpha}}}\right) \sqrt{1-\frac{U_{k}}{U_{p}}} \cdot\left(48 \sigma_{z}-0.5\right) \cdot \frac{1.4}{1-v}
\end{gathered}
$$

where $t_{k}$ is the running hours of the component from the beginning of its operation or recovery of operation after the repair up to the instant of control; $U_{p}$ is the limiting deviation of the diagnostic parameter:

$$
\mathrm{U}_{\mathrm{p}}=\left|\mathrm{P}_{\mathrm{p}}-\mathrm{P}_{\mathrm{n}}\right|-\Delta \mathrm{P},
$$

where $P_{n}$ is the nominal value of the parameter; $P_{p}^{n}$ is the limiting value of the parameter; $\Delta P$ is the characteristics of the parameter variation during the running-in interval; $\mathrm{U}_{\mathrm{k}}=\left|\mathrm{P}_{\mathrm{k}}-\mathrm{P}_{\mathrm{n}}\right|-\Delta \mathrm{P}$ is the deviation of the parameter at the running hours $t_{k} ; P_{k}$ is the measured value of the parameter at the running hours $t_{k} ; t_{M}$ is the running hours between inspections, that is, the running hours between similar types of maintenance and repair; $t_{a v}$ is the average lifetime according to the parameter; $\sigma_{\mathrm{z}}$ is the mean-square deviation of the actual variation of the parameter from the approximating function, regulated in parts of the measured deviation of the parameter; $v$ is the factor of the lifetime variation.

When $\sigma_{\mathrm{z}} \leq 0.03 \mathrm{U}_{\mathrm{p}}$, the correction factor $K_{t}$ can be eliminated of consideration, that is, we take $K_{t}$ to be equal to the unity $\left(K_{t}=1\right)$.
The experimental check-in and industrial aprobation of the proposed technique has been implemented by the experts of the Izhevsk State Technical University laboratory of the reliability problems of paper-making equipment. On the basis of the performed investigations, the following values has been ontained: the degree index of the approximating function $\alpha$ for ball bearings is 1.5 , for roller bearings it is 1.4. Analysis of results of the residual lifetime calculation showed the practically acceptable deviations on actual values and reasonability of applying the technique in order to estimate the residual lifetime of mechatronic systems and modules components with monotonous variation of the diagnostics parameter. The process of the decision making on putting the mechatronic system out of operation to repair it on the basis of estimating the residual lifetime of its component is presented by the algorithm in Figure 9 .

The parameter or a number of parameters is chosen for each unit or part, according to which it is possible to determine unambiguously the technical state of the diagnosed element. The process of obtaining the diagnosed parameter $P$ for a specific part or unit is the implementation of one of the diagnosed methods. The boundary of the area is established for each of the parameters, where the system module is accepted as serviceable. Fi- 
nally, according to the values obtained in diagnostics, the conclusion is made on the technical state of this element and on the possibility or impossibility of its further operation by comparing these values with the assigned boundaries of the operability area $O_{r a b}$. As for operable components of the system, the value of the residual lifetime is predicted. The obtained value of the residual lifetime is compared with a certain minimum value of the residual lifetime $t_{\text {min }}$, which has been determined and put into the database for each specific unit. If the calculated value is less than the minimum one, then a breakage is possible in further operation, that is why it is necessary to perform the repair of replacement of the unit, which worked out its lifetime. As for inoperable parts and parts which worked out their lifetime, the decision is made on the term of eliminating the faults. As for units and parts being recovered, the cost of maintenance and repair is calculated, and the cost of replacement is calculated for unrecoverable units. On the basis of the taken decision and according to the system of maintenance and repair, the repair and recovery measures on eliminating the failure are taken.

The corrections are put into the developed graph of repairs (applying the described above technique) according to the following algorithm. The operation time of the diagnosed equipment or components is calculated from the instant of control to the nearest planned stoppage (maintenance and repair, scheduled predictive repair and so on):

$$
t_{O P}=D_{P}-D_{\text {tech }}
$$

where $D_{P}$ is the date and time of the planned stoppage; $D_{\text {tech }}$ - date and time of performing the technical state diagnostics of the equipment or its component. Comparing the values $t_{O P}$ and $t_{O S T}$, further activities are determined. If $t_{O S T}<t_{O P}$ and the failure consequences lead to the emergency, then the date and time of the residual lifetime working out is defined and the time of the scheduled stoppage is changed. The content of the scheduled works is corrected for a new date. In case of non-criticality of the component failure, its replacement is performed during the scheduled stoppage. One should note, that it is reasonable to subject such component parts to diagnostics, since they can be used up to the failure.

In case $t_{O S T}>t_{O P}$ the multiplicity relation is calculated:

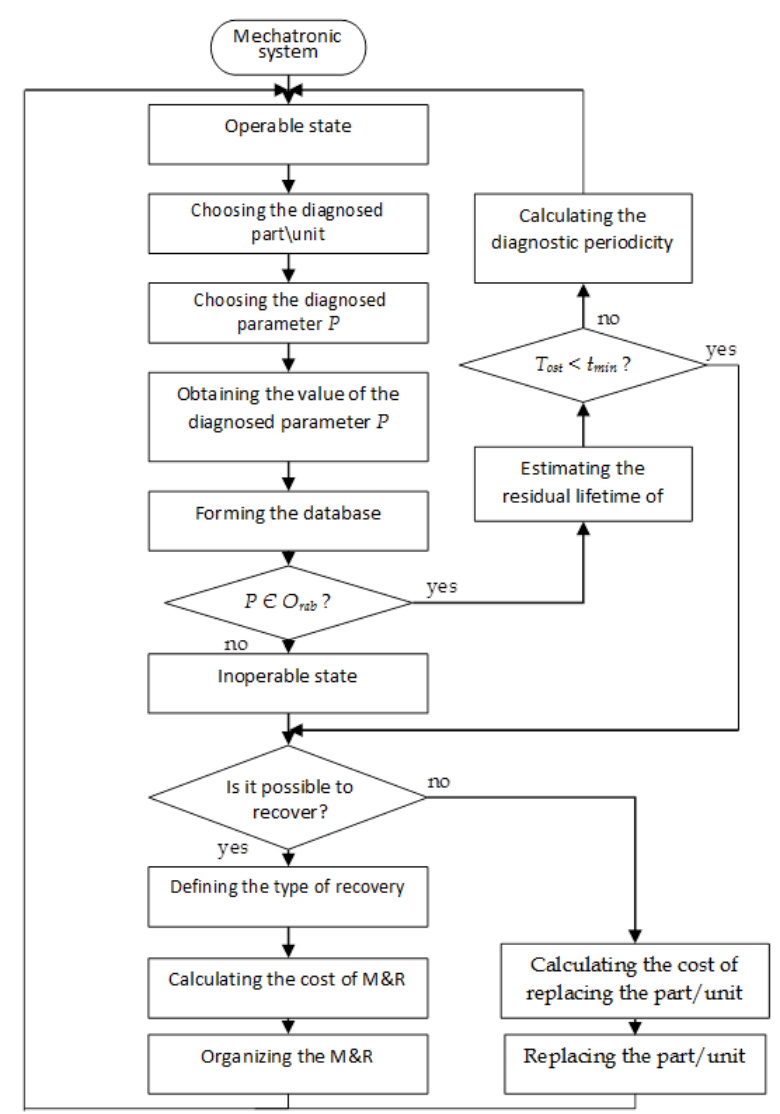

Fig. 9. The algorithm of making the decision on putting the mechatronic system out of operation to repair it, applying the diagnostics of its components technical state

$$
\frac{t_{O S T}-t_{O P}}{t_{\gamma \min }}=\hat{\mathrm{E}}_{1}
$$

where $t_{\gamma \min }$ is the minimum gamma percentile running time up to the replacement or repair of the system component, determining the periodicity of the scheduled repair and recovery works.

If $K_{1}<1$, then the repair or replacement of the diagnosed component part is performed during the nearest scheduled repair of the system. When $K_{1}>1$, the duration of operation (lifetime) up to the next diagnostics is calculated.

For example, the distribution of error-free running hours of rolling bearings, applied as shaft supports of mechatronic systems, is subjected, as a rule, to the Weibull law [8].

Then the intensity of failures for a group of single-type bearings will be written as:

$$
\lambda_{j}(t)=\frac{b_{j}}{a_{j}}\left(\frac{t}{a_{j}}\right)^{b_{j}-1}
$$


where $b$ and $a$ are parameters of the shape and scale of the Weibull distribution, correspondingly; $t$ is the considered time interval.

The periodicity of the diagnosis statement of $j^{\text {th }}$ dimension type bearing is:

$$
t_{D t} \leq 1 / \lambda_{j}(t)
$$

For a group of bearings or for the whole integrity of the machine bearings the intensity of failures will be determined as:

$$
\lambda_{\Sigma}(t)=\sum_{j=1}^{n} \lambda_{j}(t)
$$

where $n$ is the number of bearings dimension types.

In case of applying other distribution laws of the components running hours up to the failure, the technique of defining the diagnostic periodicity is the same, and the intensity of failures is calculated according to the corresponding relations.

Therefore, the presented algorithm allows introducing the corrections into the graph of the equipment maintenance and repair according to its actual technical state.

\section{Diagnosing the technical state and scheduling the maintenance and repair of complex mechatronic system}

As for the complex mechatronic systems, the task of the individual prediction of their technical state and reliability becomes urgent. Solution of this task allows not only to estimate the reliability of a specific unit of the equipment, but to pass from the scheduled and lifetime maintenance and repair to planning the operation according to the actual state, on the stipulation that advanced diagnostic equipment is available.

Defining the technical state of complex machines is one of the most sophisticated tasks in the domain of mechanical systems operation. The features of the technical state can be qualitative and (or) quantitative characteristics of these features. The actual values of these characteristics define the technical state of the object.

Increasing the intensity of systems application can be performed on acquisition of the objective data on their actual technical state. The successful solution of this task can be found only by advanced scientific methods of technical diagnostics. The result of technical diagnostics is the conclusion on the object technical state with specification (if needed) of the location, type and reason of defects $[4,15,16,17,18]$.
The application of advanced diagnostics means allows to increase the error-free running hours of separate components and systems as a whole. The strict compliance of the specified inter-repair lifetime tp for all, without exception, components independently on the technical state of each of them, does not provide the absence of failures and it leads to the untimely replacement of the majority of the system elements. In this connection the estimation of the components technical state beyond the boundaries of tp by means of technical diagnostics can considerably increase their lifetime and operation effectiveness of the paper-making machine.

The structure of the object and structural parameters. The object of investigation in the technical diagnostics is real technical objects (objects of diagnostics). Investigating the diagnostic objects covers two aspects: studying the properties and characteristics of real mechatronic systems and methods of their mathematical models definition.

The first aspect is related to the solution of the following tasks: studying the process of normal operation of the system; specifying the system elements and relations between them, that is, the structure of the system; specifying the possible states of the system, that is, the possible failures of elements; analyzing the technical possibilities of controlling the features, characterizing the system state; accumulation and processing the statistical data, allowing to define the probability distribution of possible states of the system, laws of failures occurrence for separate elements and costs related to diagnostics. All these tasks solutions imply the empirical investigation of specific technical systems and procedures of their diagnostics.

The second aspect is related to definition of the mathematical models of the objects. The theoretical analysis always implies a definite idealization, when certain features, essential for the technical diagnostics, are singled out and minor features are eliminated, that is, a real technical system is replaced by a certain model. This model should be rather formalized and generalized to be accepted for the analysis of the whole class of technical systems. At the same time it should take into account all the essential features of specific systems and methods of searching the failed elements there. Replacing the real components by corresponding models allows to apply widely the formal means of the modern mathematics in order 
to solve the technical diagnostic tasks (probability theory, mathematical statistics, mathematical logics, dynamic programming, and so on.

The object of diagnostics (mechatronic systems and modules) has a definite structure, that is, a well-ordered totality of complexes of operating jointly elements (parts) which form the layout of the object, thus providing the performance of the assigned functions.

The system structure is characterized by mutual arrangements, shapes and dimensions of interacting parts (macrostructure), the character of conjugations, surface roughness (microstructure) and other characteristics. The structure of the object, in particular, of the mechatronic system, predefines the totality, assigned in design and production, of definite technical and economical features (manufacturability, energy, economics matters and other) as a whole. This totality, in its turn, defines the level of adaption of the object to perform certain functions at definite operation conditions.

The object structure is characterized by quantitative parameters, which are called structural. Mechatronic systems, coming to operation, possess a certain structure and totality of technical and economical features, dependent on the structure. Numerical values of structural parameters correspond here to the values, assigned according to drawings and technical specifications. Such values of parameters are called initial or nominal.

The macrostructure remains constant in the operation process. The number and mutual arrangement of parts and units in a specific object are constant, but their interactions in conjugations of separate parts (microstructure) are varied due to wearing out and other destruction processes (accumulation of damages). When the object microstructure is changed in parts conjugations, the character of their interaction is also changed. Considering the variation of the object structure, one can judge on its technical state at each assigned time instant, characterized by a certain totality of specific values of structural parameters of parts and units. Variation of the object structural parameters influences the totality of its technical operational features and it has quite definite laws, which have not been studied profoundly yet. When accumulating constantly, these variations can reach such a quantitative value (limit), when uneven variations occur (for example, destruction of parts, preceded by the appearance of micro-cracks in the material) and the failure takes place.
All faults and failures, appearing in operation of mechatronic systems, modules and their components, are accompanied by variation of clearances and interferences in conjugations, wear, fatigue pitting, noise, vibrations, temperature modes violation, functional characteristics variation and so on. These features, which accompany faults and failures, can be the basis of technical state evaluation and can often be estimated quantitatively.

Diagnostic parameters, characterizing the object technical state, can be structural (direct) and indirect. Structural parameters (for instance, wear, clearance in conjugation, pitting of the material surface and so on) immediately specify the serviceability and technical state of the equipment components under control.

In practice, the parameters are applied, meeting the requirement of unambiguity, variation range, accessibility and convenience of measurement, information value and manufacturability. The parameters are first taken into account, which characterize the most frequently repeating failures and faults or which are very expensive to eliminate.

If the parameters of the system or its components are within the limits, regulated by the reference technical documentation (they belong to the area of allowable values), then the state of the object is considered as operable. Going out of the boundaries of allowable values is considered as the failure, and the properties of the system to keep in time all the parameters within the stated limits, which in turn characterize the ability to perform the required functions at the modes and conditions of operation, define the reliability of this operation. In this connection such important characteristics of the system, as technical state, serviceability and reliability are associated with the concepts of the parameter and the process of the parameter variation.

\section{RESULTS AND EVALUATION OF CHANGES}

Maintenance according to the repair is based on the estimation of the investigated object technical state according to the data on controlling its parameters and predicting their variation. The individual prediction of the technical state allows to use the controlled object till the dangerous reduction of serviceability (failures appearance), to eliminate the untimely intervention in its operation and performing labor-intensive maintenance operations, which are often not very useful from the operation reliability point of view. 
Analyzing the stated above, one can define the role of the technical diagnostics in the system of M\&R as follows: the advanced diagnostic assurance of the mechatronic system operation process is the integral condition of passing to the predictive maintenance and repair.

Requirements to the diagnostic assurance (technical diagnostics) of the mechatronic system operation process with regard to the system of M\&R can be defined as follows: defects, the intensity of their development and the technical state of the diagnosed component should be detected unambiguously and at a high reliability level; variation of diagnostic parameters should be predicted and the value of the residual lifetime of the component should be determined with the assigned degree of risk; the diagnostic assurance should be reliable in operation; it should provide the periodicity of performing the diagnostics in order to prevent the emergency situations; it should ensure the possibility to measure several diagnostic parameters for an objective and reliable estimation of the object technical state; it should signal when the diagnostic parameter reaches its allowable value and the prior-to-failure state; it should provide the accumulation and storage of information in variation of diagnostic parameters in the operation process with regard to each component part; it should have the prospects of expansion and enhancement (development); and it should ensure the efficient diagnostics.

Meeting all the above enumerated requirements will provide the effectiveness of technical diagnostics of the components and the system of maintenance and repair of mechatronic systems as a whole due to the reduction of the unscheduled stoppages in the repair and of the repair works content at the more efficient coverage of the components lifetime.

\section{CONCLUSION}

The following results have been obtained during the performed investigation:

- Models of mechatronic systems operation and reliability have been obtained, allowing to predict the critical situations according to the stated criteria.

- Backgrounds of constructing the mechatronic system repair schedule on the basis of estimating the reliability level (statistical analysis) of component parts have been proposed. The considered above principles and algorithms of defining the content of repair on the basis of running hours multiplicity of the machine elements increase the operation effectiveness of the equipment.

- Semi-Markovian representations of the operation and reliability model implement the concept of a specific mechatronic system effectiveness. The possibility to implement the concept is explained by the independence, uniqueness and complexity of the mechatronic system.

- Development of a flexible adaptive strategy of the repair organizing with account of the technical state and reliability level of the design elements will provide the operation with account of the state and the highest operation effectiveness of the mechatronic system.

- Requirements to diagnostic assurance of mechatronic systems operation process with regard to the system of maintenance and repair, ensuring the process effectiveness, have been determined.

The obtained results create the background for the development of the automated system of maintenance and repair of mechatronic systems and modules on the basis of their technical state estimation.

\section{ACKNOWLEDGEMENTS}

The reported study was funded by RFBR according to the research project № 18-08-00772 A. The contribution is sponsored by the project $015 \mathrm{STU}-4 / 2018$ Specialised laboratory supported by multimedia textbook for subject "Production systems design and operation" for STU Bratislava.

\section{REFERENCES}

1. Abramov I.V. and Turygin Yu.V. Operation efficiency and strategies of maintenance and repair of paper-making machines. Proceedings of the international conference Problems of system support of industrial production quality, Izhevsk, Russia 1997, 3-6.

2. Abramov I.V., Turygin Yu.V., and Komarov K.L. Development of the repair cycle control model of continuously working complex systems according to statistical data. Methods of computational experiment in engineering practice, 2, 1992, 126-129.

3. Abramov I.V., Turygin Yu.V. and Nikitin Yu.R. Model of equipment operation on the basis of technical state diagnostics, Methods of computational experiment in engineering practice, 3, 1992, 119-124. 
4. Grămescu, B. and Niţu, C. Parameter identification of a DC servomotor. Romanian Review Precision Mechanics, Optics and Mechatronics, 50, 2016, 227-230.

5. Božek P. Robot path optimization for spot welding applications in automotive industry. Tehnički Vjesnik - Technical Gazette, 20(5), 2013, 913-917.

6. Eryemin M.S., Abramov I.V. Abramov A.I. and Turygin Yu.V. Strategy of predictive machines repair. Repair, recovery, modernization, 1, 2011, 20-24.

7. Klyagin V.I. and Sabirov F.S. Typical maintenance and repair system of metal- and wood-working equipment. Mashinostroyeniye, Russia.

8. Kugel R.V. and Borisov Yu. S. Regulated distributions of machine elements lifetime. Vestnik mashinostroyeniya, 10, 1976, 7-10.

9. Mikhlin V.M. Reliability control of agricultural equipment, Kolos, Russia.

10. Liu, R., Yang, B., Zio, E. and Chen, X. Artificial intelligence for fault diagnosis of rotating machinery. Mechanical Systems and Signal Processing, 108, 2018, 33-47.

11. Turygin Yu.V., Maga D. and Faitova N. Building of a flexible (adaptive) structure for mechatronic system's repair cycle. Proceedings of 13th International Conference on Mechatronics - Mechatronika 2010, Trencianske Teplice, Slovakia 2010, 112-114.

12. Turygin Yu.V. and Sychev A.A. Technique of repair cycle correction of paper-making equipment on the basis of technical state diagnostics. Proceedings of the scientific technical conference Scientists of Izhevsk technical university - to the production, Izhevsk, Russia 1990, 68.

13. Turygin Yu., Božek P., Nikitin Yu., Sosnovich E. and Abramov A. Enhancing the reliability of mobile robots control process via reverse validation. International Journal of Advanced Robotic Systems, 2016, 1-8.
14. Abramov I.V., Nikitin Y., Abramov A.I., Sosnovich E. and Božek P. Control and diagnostic model of brushless DC motor. Journal of Electrical Engineering, 65(5), 2014, 277-282.

15. Božek P. and Pivarčiová E. Registration of Holographic Images Based on Integral Transformation. Computing and Informatics, 31(6), 2012, 1369-1383.

16. Abramov I., Božek P., Abramov A., Sosnovich E. and Nikitin Y. Diagnostics brushless DC motors. Proceedings of the 55th Conference on Experimental Stress Analysis, 2017, 156-164.

17. Stepanov P., Lagutkin S. and Nikitin Y. Development of the Algorithm for Definition of Residual Service Life by a Comprehensive Diagnosis of the Electromechanical Drive. Acta Mechanica Slovaca, 19(2), 2015, 52-56.

18. Nikitin Y.R., Abramov I.V., Turygin Y.V. and Abramov A.I. Diagnostics of mechatronic systems. Acta Mechatronica, 1(1), 2016, 9-112.

19. Dębski H., Wysmulski P. and Falkowicz K. A study of stability and post-critical behaviour of thin-walled composite profiles under compression. Eksploat. I Niezawodn. - Maint. Reliab., 18, 2016, 632-637.

20. Grega R., Krajňák J., Žul'ová L., Fedorko G. and Molnár V. Failure analysis of driveshaft of truck body caused by vibrations. Eng. Fail. Anal., 79, 2017, 208-215.

21. Garbacz T., Jachowicz T., Gajdoš I. and Kijewski G. Research on the Influence of Blowing Agent on Selected Properties of Extruded Cellular Products. Adv. Sci. Technol. Res. J., 9 (28), 2015, 81-88.

22. Žul'ová L., Grega R., Krajňák J., Fedorko G. and Molnár V. Optimization of noisiness of mechanical system by using a pneumatic tuner during a failure of piston machine. Eng. Fail. Anal., 79, 2017, 845-851. 